\title{
Upregulated LINC01088 Facilitates Malignant Phenotypes and Immune Escape of Colorectal Cancer by Regulating microRNAs/G3BP1/PD-L1 axis
}

\section{Shukui Wang ( $\nabla$ sk_wang@njmu.edu.cn )}

Southeast University Medical College https://orcid.org/0000-0001-6972-2587

\section{Chenmeng Li}

Southeast University Medical College

\section{Bei Pan}

Southeast University Medical College

\section{Xuhong Wang}

Southeast University Medical College

Jian Qin

Southeast University Medical College

\section{Xiangxiang Liu}

Southeast University Medical College

\section{Tianyi Gao}

Nanjing Medical University affiliated Nanjing Hospital: Nanjing First Hospital

\section{Huiling Sun}

Nanjing Medical University affiliated Nanjing Hospital: Nanjing First Hospital

\section{Yuqin Pan}

Nanjing Medical University affiliated Nanjing Hospital: Nanjing First Hospital

\section{Research Article}

Keywords: LINC01088, microRNAs, PD-L1, immune escape, colorectal cancer

Posted Date: January 18th, 2022

DOI: https://doi.org/10.21203/rs.3.rs-1259309/v1

License: (c) (1) This work is licensed under a Creative Commons Attribution 4.0 International License. Read Full License 


\section{Abstract}

Purpose: Long intergenic non-coding RNA LINC01088 is a newly discovered long non-coding RNA. Its biological function in colorectal cancer (CRC) remains unknown.

Methods: Here, 36 paired CRC and para-cancerous tissues were collected. In vitro, fluorescence in situ hybridization assay, qPCR, western blotting analysis and cellular functional experiments, RNA immunoprecipitation (RIP) assay and dual-luciferase reporter system analysis were performed. In vivo, xenograft tumor mouse models were generated. Besides, patient-derived intestinal organoid (PDO) was used ex vivo.

Results: We found that LINC01088 was significantly upregulated in colorectal cancer tissues and CRC cell lines compared to adjacent normal tissues and colonic epithelial cells. High LINC01088 expression was associated with poor outcome in CRC. LINC01088 was mainly located in the cytoplasm. LINC01088 knockdown suppressed the proliferation, migration, invasion, and immune escape of colorectal cancer cells. Mechanistically, LINC01088 bound directly to miR-548b-5p and miR-548c-5p that were significantly upregulated G3BP1 expression, altering CRC cell phenotypes. In mouse xenograft models, LINC01088 knockdown restrained tumor growth and lung metastasis. Further, G3BP1 overexpression reversed LINC01088-knockdown mediated inhibitory effects on tumor growth. Notably, LINC01088 knockdown downregulated PD-L1 expression, while G3BP1 overexpression restored PD-L1 expression in xenograft tumors. Besides, LINC01088 knockdown repressed CRC organoid growth ex vivo.

Conclusion: Overall, these findings suggested that LINC01088 directly targeted miR-548b-5p and miR548c-5p promoting G3BP1 and PD-L1 expression, which facilitated colorectal cancer progression and immune escape.

\section{Introduction}

Colorectal cancer (CRC) remains a serious threat to human health globally, with nearly two million new cases and approximately one million deaths caused each year (Dekker et al, 2019; Sung et al, 2021). Although the early diagnosis and clinical treatment of CRC have been greatly advanced in the last decade (Anania et al, 2019; Van Cutsem et al, 2014), it continues to be a significant burden on the individual and society. Recent molecular targeted therapy and immunotherapy are considered to be scientific breakthroughs in treatment of tumors including colorectal cancer (Franke et al, 2019; Nappi et al, 2018). Nevertheless, not all patients benefit from it. There are still unknown mechanisms in colorectal cancer development and progression. Hence, it is necessary to explore molecular mechanisms of CRC.

Long non-coding RNAs (IncRNAs) with a length of more than 200 nucleotides are a class of non-coding RNAs discovered in recent years including antisense long non-coding RNAs (antisense IncRNAs) and long intronic non-coding RNAs (lincRNAs) (Quinn \& Chang, 2016; Ransohoff et al, 2018). Previously these noncoding RNAs were considered as a large class of "junk" RNA produced during transcription, until recent studies have identified that they have important biological functions (Palazzo \& Koonin, 2020; Quinn \& 
Chang, 2016). And growing evidences support that long non-coding RNAs engage in a variety of physiological or pathological processes including organismal development and cancer (Dykes \& Emanueli, 2017; Fang \& Fullwood, 2016; Gutschner \& Diederichs, 2012; Sanchez Calle et al, 2018; Schmitz et al, 2016). We recently reported that several IncRNAs are implicated in colorectal cancer progression (Li et al, 2021; Xu et al, 2019; Zhou et al, 2021), of which downregulated LncRNA SATB2-AS1 expression is associated with poor outcome in CRC patients. LncRNA SATB2-AS1 in the nucleus binds directly to WDR5 and GADD45A, and cis-activates SATB2 (specific AT-rich binding protein 2) transcription via regulation of histone $\mathrm{H} 3$ lysine 4 trimethylation (H3K4me3) deposition and DNA demethylation in the SATB2 promoter region, inhibiting colorectal cancer metastasis and affecting tumor immune cell microenvironment (Xu et al., 2019). In addition, a recent study has reported that other long non-coding RNAs localized in the cytoplasm, such as LncRNA-ATB and Linc00284, are able to bind to microRNAs (miRNAs) and cause dysregulation of miRNAs expression ( $\mathrm{Li}$ et al, 2020; You et al, 2021). Linc00284 is highly expressed in $\mathrm{CRC}$ tissues and CRC cells and associated with tumor metastasis. Linc00284 regulates miR-27a/c-Met axis contributing to activation of downstream signaling pathways that result in malignant phenotypes of CRC cells (You et al., 2021).

The role of long non-coding RNAs in the development and progression of various cancers including colorectal cancer, is becoming clearer with the progressive research (Chi et al, 2019; Sanchez Calle et al., 2018), but with the increasing number of newly discovered IncRNAs, it is essential to understand precise mechanisms of these novel IncRNAs in cancer. LINC01088 located on Chromosome 4 at q21.21 is a newly discovered long non-coding RNA that has recently been investigated. A bioinformatic analysis of two GEO datasets (GSE28619 and GSE143318) identified that LINC01088 was down-regulated in liver tissues from patients with acute alcoholic hepatits compared to samples derived from donor livers (Yan et al, 2021). However, another analysis of TCGA database demonstrated that LINC01088 was significant highly-expressed risk candidate in primary lung squamous cell carcinoma compared with adjacent normal tissues and predicts poor prognosis (Liu et al, 2019). In addition, work in non-small cell lung cancer (NSCLC) indicated that LINC01088 was overexpressed in NSCLC tissues and cell lines, where LINC01088 promotes cancer cell proliferation via binding with EZH2 to repress p21 (Liu et al, 2020). Conversely, in ovarian cancer (OC), LINC01088 expression was markedly lower in OC tissues in comparison to adjacent noncancerous tissues, which is an independent predictor for overall survival (Ai et al, 2018; Zhang et al, 2018). These evidences suggest that the expression pattern of LINC01088 can be different in different types of diseases. In our previous screen, it was found to be upregulated in CRC tissues and was associated with multiple clinicopathological features in colorectal cancer patients. Herein, we investigated the role of LINC01088 in CRC with the aim of gaining a deeper understanding of colorectal cancer progression and providing potential target for CRC diagnosis and treatment.

\section{Materials And Methods}

\section{Colorectal cancer tissues}


Thirty-six cancer tissues and paired tissues were collected from CRC patients with complete pathological and clinical data who were hospitalized and treated surgically in Nanjing First Hospital from January 2015 to January 2020. Adjacent tissue was taken from a distance of more than $5 \mathrm{~cm}$ from cancerous tissue, and intraoperative pathological slices were pathologically confirmed to be free of cancer. There were 21 males and 15 females, aged (61.6 \pm 7.7$)$ years, with an age range of 51-72 years. Inclusion criteria: 『surgically resected specimens were pathologically confirmed; खall patients with colorectal cancer did not receive anti-cancer treatment such as chemotherapy, radiotherapy, immunotherapy, and targeted therapy before surgery; 『clinical and pathological data of CRC patients were complete; खall patients gave informed consent. Besides, CRC patients with other malignant tumors, systemic infections, immunodeficiency, immune diseases, coagulation dysfunction, serious heart, liver, or kidney diseases were excluded. All patients signed informed consent form prior to surgery, and the study was approved by the Ethics Committee of Nanjing First Hospital. Table 1 lists patient characteristics.

Table 1

The clinicopathological characteristics of patients with colorectal cancer (36 cases).

\begin{tabular}{|ll|}
\hline Characteristics & $\begin{array}{l}\text { Number of cases (\%) } \\
\text { of cases[\%] }\end{array}$ \\
\hline Age (year) & $15(41.67)$ \\
\hline$\square 60$ & $21(58.33)$ \\
\hline Gender & $17(47.22)$ \\
\hline Male & $19(52.78)$ \\
\hline Female & $20(55.56)$ \\
\hline TNM stage & $16(44.44)$ \\
\hline$\square+\bigotimes$ & \\
\hline$\square$ & $17(47.22)$ \\
\hline Recurrence & $19(52.78)$ \\
\hline No & $24(66.67)$ \\
\hline Yes & $12(33.33)$ \\
\hline Distant metastasis & \\
\hline M0 & \\
\hline M1+M2 & \\
\hline
\end{tabular}




\section{Cell culture and lentivirus infection}

Human colorectal cancer cell lines LoVo, Caco2, HCT116, SW480, normal human colon epithelial cells CCD841CoN, and 293T cells were obtained from the American Type Culture Collection (ATCC). LoVo cells were maintained in Ham's F-12 culture medium with 10\% FBS (Gibco) and 1\% penicillin/streptomycin (P/S). Caco2, HCT116, SW480 and 293T cells were grown in Dulbecco's modified Eagle's medium (DMEM) (Gibco) containing 10\% FBS and 1\% P/S. CCD841CoN cells were cultured in minimum essential medium (MEM) with $10 \% \mathrm{FBS}$ and $1 \% \mathrm{P} / \mathrm{S}$. All cells were kept in $5 \% \mathrm{CO}_{2}$ incubator at $37^{\circ} \mathrm{C}$. LINC01088 silencing lentivirus (sh1-01088 and sh2-01088), G3BP1-overexpressing lentivirus and the control lentivirus were constructed by Shanghai GeneChem Co., Ltd. (China). CRC cells were infected with shRNA lentivirus or/and G3BP1-overexpressing lentivirus or control lentivirus at $10 \mathrm{MOI}$ for $24 \mathrm{~h}$ following 7-days puromycin $(2 \mu \mathrm{g} / \mathrm{ml})$ selection. LINC01088 knockdown efficiency in the infected cells was validated by qRT-PCR.

\section{Fluorescence in situ hybridization (FISH) assay}

Fluorescence in situ hybridization (FISH) assay was conducted using RNA FISH Kit (Shanghai GenePharma Co., Ltd, China) according to the manufacturer's protocol. All sequences used were listed below. 5'-Cy3-labeled LINC01088 homo probe 1 (5'-3'): GCAGTACTAACTCATCCAGTATCTGATTC; 5'-Cy3labeled LINC01088 homo probe 2 (5'-3'): GGCGGCAGCAAGAAGCAGTTCTAAT; 5'-Cy3-labeled LINC01088 homo probe 3 (5'-3'): CTTTGCATCCATCATTCAACGAAAT; 5'-FAM-labeled 18S (5'-3'):

CTGCCTTCCTTGGATGTGGTAGCCGTTTC; 5'-FAM-labeled U6 (5'-3'): TTTGCGTGTCATCCTTGCG; 5'-Cy3labeled negative control sequence (5'-3'): TGCTTTGCACGGTAACGCCTGTTTT.

\section{Transfection experiments}

Small interfering RNA (siRNA) oligonucleotides including the control scrambled siRNA were ordered from Sangon Biotech (Shanghai) Co., Ltd. (China). Transient transfection of siRNA was performed with Lipofectamine $^{\text {TM }}$ RNAiMAX Transfection Reagent according to manufacturer's instructions (Invitrogen, cat no.13778500). The RNA-lipid complexes were prepared, vortexed and allowed to incubate for $5 \mathrm{~min}$ at room temperature (RT). Next, the mixture was added into the cells followed by incubation for $24 \mathrm{~h}$ at 37 ${ }^{\circ} \mathrm{C}$. The efficiency of siRNA interference was determined by qRT-PCR analysis 48 hours post-transfection.

\section{Nucleo-cytoplasmic separation experiment}

Nucleo-cytoplasmic fractions were isolated using PARIS ${ }^{\mathrm{TM}}$ Kit following manufacturer instructions. Briefly, adherent cells ( $10^{7}$ cells per experiment) were harvested until they were grown to $90 \%$ confluence, transferred to $1.5 \mathrm{~mL}$ microfuge tubes, and centrifuged at $180 \times \mathrm{g}$ for 4 min at $4{ }^{\circ} \mathrm{C}$. Next, the 
supernatant was discarded. $500 \mu \mathrm{l}$ of ice-cold Cell Fractination Buffer was added. Tubes were then placed on ice for $7 \mathrm{~min}$. After centrifugation at $500 \times \mathrm{g}$ for $4 \mathrm{~min}$ at $4{ }^{\circ} \mathrm{C}$, the supernatant (cytoplasmic fraction) was transferred to a new RNase-free $1.5 \mathrm{~mL}$ EP tube. The pellet contained nuclear fraction. Total nuclear or cytoplasmic RNA was extracted, respectively. RNA samples were stored at $-80^{\circ} \mathrm{C}$ or kept on ice when in use.

\section{Cell Counting Kit-8 (CCK-8) assay}

The cells were inoculated into 96 -well dishes at a density of $3 \times 10^{4}$ cells per well, and five replicate wells were set up in each analysis. After $24 \mathrm{~h}, 48 \mathrm{~h}, 72 \mathrm{~h}$ and $96 \mathrm{~h}$ of incubation respectively, CCK-8 reagent (Sigma-Aldrich, cat no.96992) with a volume fraction of $10 \%$ was added to the cells following incubation for $2 \mathrm{~h}$ at $37^{\circ} \mathrm{C}$. The plates were placed on a shaker for $5 \mathrm{~min}$, and absorbance values were measured at $490 \mathrm{~nm}$ using Microplate reader (Bio-Rad). Growth curve was plotted with time as the horizontal coordinate and absorbance value as the vertical coordinate.

\section{Colony-formation assay}

The cells (500 cells per well) were inoculated into 6-cm culture dishes. After 2 weeks of incubation at 37 ${ }^{\circ} \mathrm{C}$, cell colonies were observed and then culture medium was discarded. Adherent cells were gently washed twice with PBS (Gibco) and fixed with $4 \%$ paraformaldehyde for $15 \mathrm{~min}$ at RT. After elimination of fixative solution by aspiration, cell colonies were visualized by staining with $0.1 \%$ crystal violet solution (Beyotime Biotechnology, cat no.C0121, China) for 20 min at RT. The number of colonies was counted.

\section{Cell cycle analysis}

Cell cycle was evaluated by flow cytometry. The cells in logarithmic growth phase were digested using EDTA-free trypsin (Gibco) and washed three times with pre-chilled PBS. Thereafter, $400 \mu$ of Binding Buffer and $10 \mu \mathrm{l}$ of PI were added sequentially to cell suspension, following reaction for 30 min under protection from light at RT. Cycle distribution was assessed using CytoFlex Flow Cytometer (Beckman Coulter).

\section{Transwell migration and invasion assays}

For transwell invasion assay, Matrigel (Corning, cat no.356234) was removed from the $-20^{\circ} \mathrm{C}$ refrigerator and placed in an ice-water mixture to dissolve. All tips and microfuge tubes used in the experiments were pre-chilled in a $4^{\circ} \mathrm{C}$ refrigerator. Matrigel and serum-free basal medium were mixed thoroughly at a 1:3 ratio and then added into transwell upper chambers (Costar, 8- $\mu \mathrm{m}$ pore size) for incubation for $1 \mathrm{~h}$ at $37^{\circ} \mathrm{C}$. In addition, cell concentration was adjusted to $5 \times 10^{5} \mathrm{cell} / \mathrm{s} / \mathrm{ml} .200 \mu \mathrm{l}$ of the cell suspension was slowly added to the upper chambers and the lower chambers contained $500 \mu$ l of complete culture 
medium. Twenty-four hours later, the non-invasive cells in transwell upper chamber were wiped off with wet cotton swabs. Next, the filter was fixed with $4 \%$ formaldehyde for $10 \mathrm{~min}$, stained with $0.5 \%$ crystal violet for 20 min, rinsed with PBS and air-dried. The number of invasive cells was observed microscopically, and 10 randomly chosen fields of view were imaged and counted. Transwell chambers pre-coated without Matrigel were used for transwell migration assay and workflow was followed as indicated above.

\section{Propidium iodide $(\mathrm{PI})$ staining}

After $48 \mathrm{~h}$ of co-culture, the cells were incubated with PI staining solution (Sigma) for $5 \mathrm{~min}$. Next, PIpositive cells were observed and counted under a fluorescent microscope (Nikon), while the total number of cells was observed under white light. The percentage of PI-positive cells was calculated.

\section{Dual-luciferase reporter assay}

The pmirGLO dual-luciferase vectors and dual-luciferase reporter system (Promega) were used for evaluating the interaction between LINC01088 and microRNAs according to the manufacturer's instructions. Predicted binding sites were analyzed by https://starbase.sysu.edu.cn/starbase2/ and http://starbase.sysu.edu.cn/. The desired primer sequences were cloned into luciferase reporter plasmid as described in the instruction manual. Empty vector plasmid was as the control. 293T cells were seeded into 24 -well plates and allowed to grow to $80 \%$ confluence. Reporter plasmid was co-transfected with the expression plasmid into 293T cells using Lipofectamine ${ }^{\mathrm{TM}} 2000$ Reagent (Invitrogen). Seventy-two hours later, the proteins were extracted and Dual-Luciferase Reporter Assay System Kit (Promega) was used to test luciferase activity.

\section{RNA immunoprecipitation (RIP) assay}

RIP assay was performed using Imprint ${ }^{\circledR}$ RNA Immunoprecipitation Kit according to the manufacturer's instructions (Sigma-Aldrich). Briefly, CRC cells with LINC01088 knockdown in logarithmic growth phase were harvested and resuspended using RIP Lysis Buffer, mixed thoroughly, and set aside on ice. After incubation for $30 \mathrm{~min}$, the samples were centrifuged at $2500 \times \mathrm{g}$ for $10 \mathrm{~min}$ at $4^{\circ} \mathrm{C}$. Besides, magnetic beads conjugated anti-Ago antibody or IgG for pre-clearance were washed with RIP Wash Buffer (provided with the kit). $900 \mu \mathrm{l}$ of RIP Immunoprecipitation Buffer was added to each tube and mixed with $100 \mu$ supernatant following incubation overnight at $4^{\circ} \mathrm{C}$ with rotation. After precipitating the magnetic beads, removed the supernatant, added $500 \mu \mathrm{l}$ of RIP Wash Buffer to wash the beads, vortexed, and then collected the precipitate. $10 \mu \mathrm{l}$ of the cell lysate supernatant was taken as "Input" and store it temporarily at $-80^{\circ} \mathrm{C}$. Next, $150 \mu \mathrm{l}$ of Proteinase K Buffer was added to the precipitate obtained in the previous step. In addition, RIP Wash Buffer, $10 \%$ SDS, and Proteinase K mixture were added to the "Input" after thawing, followed by incubation for 30 min at $55^{\circ} \mathrm{C}$ with shaking to digest the proteins. Total RNA for subsequent 
qPCR analysis was extracted from each group according to the instructions of RNA-easy Isolation Reagent (as indicated below).

\section{qPCR analysis}

Total RNA was extracted from tissues or cells using RNA-easy Isolation Reagent (Vazyme Biotech Co., Ltd, cat no. R701-01) according to the instructions and the concentration of total RNA were determined using UV spectrophotometer. cDNA was reverse transcribed using PrimeScript ${ }^{\mathrm{TM}} \mathrm{RT}$ reagent Kit with gDNA Eraser (Takara, cat no. RR047A) or miRNA 1st Strand cDNA Synthesis Kit (by stem-loop) (Vazyme Biotech Co., Ltd, cat no. MR101-01). qPCR reaction system was $20 \mu \mathrm{L}: 10 \mu \mathrm{l}$ miScript SYBR ${ }^{\circledR}$ Green Mix (QIAGEN, Dusseldorf, Germany), or $10 \mu \mathrm{l}$ miRNA Universal SYBR qPCR Master Mix (Vazyme Biotech Co., Ltd, cat no. MQ101-01), $2 \mu \mathrm{l} \mathrm{cDNA}, 1 \mu$ leach of forward and reverse primers, $6.0 \mu \mathrm{lddH} 2 \mathrm{O}$. Reaction conditions were as described below: pre-denaturation $95^{\circ} \mathrm{C}, 90 \mathrm{sec}$; denaturation $95^{\circ} \mathrm{C}, 30 \mathrm{sec}$; annealing $60^{\circ} \mathrm{C}, 30 \mathrm{sec}$; extension $72^{\circ} \mathrm{C}, 15 \mathrm{sec} ; 40$ cycles in total. Gene expression was analyzed by the $2^{-\Delta \Delta \mathrm{Ct}}$ method. GAPDH was used as an internal reference gene.

\section{Western blot analysis}

Tumor cells or tissues were subjected to RIPA lysis solution containing 1\% PMSF and placed on ice for 20 min. The supernatant was collected by centrifugation at $12000 \times \mathrm{g}$ for $15 \mathrm{~min}$ at $4^{\circ} \mathrm{C}$. Protein concentration was determined by the BCA method. Appropriate amount of protein samples was mixture with $5 \times$ loading buffer and boiled for $5 \mathrm{~min}$ to denature the proteins. SDS-PAGE gel electrophoresis was performed to separate the proteins, and $30 \mu \mathrm{g}$ of denatured protein samples were added to each lane. Proteins were transferred onto the PVDF membranes (Immobilon transfer membrane, Millipore Corporation) through wet transfer. Next, the membranes were blocked with bovine serum albumin diluted with TBST for $2 \mathrm{~h}$ at RT and incubated with primary antibody at $4{ }^{\circ} \mathrm{C}$ overnight. The secondary antibody was then added and incubated for $2 \mathrm{~h}$ at RT. Immunoblots were visualized using Immobilon Western Chemiluminescent HRP Substrate (ECL) (Millipore). $\beta$-actin protein was used as internal control.

\section{Animal experiments}

To test proliferative ability of CRC cells in vivo, tumor cells in logarithmic growth phase were resuspended in PBS and cell concentration was adjusted to $1 \times 10^{7}$ cells $/ \mathrm{ml}$. BALB/c female nude mice of age 5 weeks and weighing about $20 \mathrm{~g}$ were acclimated for one week prior to experiments. $100 \mu \mathrm{l}$ of cell suspension was subcutaneously injected into the right axilla of nude mouse ( $\mathrm{N}=6$ mice per group). Tumor diameters of xenograft mice were measured weekly after inoculation. Tumor volume was calculated by the formula: tumor volume $=$ width $^{2} \times$ length $/ 2$ Mice were sacrificed under anesthesia 35 or 42 days after inoculation, and tumor tissues were photographed and weighed after stripping and removing the surrounding connective tissues. Besides, to measure lung metastatic capability of LINC01088-knockdown CRC cells in 
vivo, CRC cell concentration of each group was adjusted to $1 \times 10^{6}$ cells $/ \mathrm{ml} .50 \mu$ of the cell suspension was injected into the mice through the lateral tail vein of the nude mice ( $\mathrm{N}=6$ mice per group) and lung metastatic nodules were examined at the desired time point after deep anesthesia with isoflurane. Lung tissues were collected for HE staining. All animal experiments were conducted under the approval of Animal Care and Use Committee of Nanjing First Hospital.

\section{Human colorectal cancer tissue-derived organoids}

This study was approved by the Ethics Committee of Nanjing First Hospital. The patient signed informed consent form prior to surgery. Fresh colorectal cancer tissues were collected after surgical resection and maintained in ice-cold DMEM/F-12 medium with 15 mM HEPES (Gibco) within 2 hours. Tissues were cut into 1-3 $\mathrm{mm}^{3}$ pieces, washed ten times using pre-chilled PBS, and digested with Gentle Cell Dissociation Reagent (GCDR) (Stemcell Technologies, cat no.07174) for 50 min at $37^{\circ} \mathrm{C}$ with shaking. After centrifugation at $300 \times \mathrm{g}$ for $5 \mathrm{~min}$, the supernatant was discarded. The tissues were resuspended using DMEM/F-12 medium with 15 mM HEPES and 2\% BSA (Solarbio Life Science, cat no.9048-46-8) and filtered through $70-\mu \mathrm{m}$ strainers. The number of intestinal crypts were counted. Next, Matrigel Matrix Growth Factor Reduced (GFR), Red-free (Corning, cat no.356231) was diluted with DMEM/F-12 complete medium (as indicated above) at a 1:1 ratio and the crypts were resuspended. Matrigel containing intestinal crypts was dropped onto the center of a pre-warmed 24-well plate without medium (50 $\mu \mathrm{l} /$ well). The plate was placed in an incubator at $37^{\circ} \mathrm{C}$ for $10 \mathrm{~min}$. Subsequently, human organoid medium IntestiCult OGM (Stemcell Technologies, cat no. A8010) was added and was changed every 2-3 days. Passaging was performed 8 days after culturing.

\section{Isolation, characterization and cultivation of human CD8+T cells}

Fresh blood from a healthy donor was transferred into a blood draw tube containing heparin anticoagulant and mixed upside down. PBS supplemented with $2 \%$ FBS (PBST) was added to dilute the blood at a 1:1 ratio. Subsequently, an equal volume of Lymphocyte Separation Solution (Solarbio Life Science, catalog \#P8610/P8900) was added to the bottom of SepMate ${ }^{T M}-50$ tube (Stemcell Technologies, catalog \#15450). The diluted blood sample was aspirated and slowly spread along the wall of the tube on top of Lymphocyte Separation Solution following centrifugation at $1200 \times \mathrm{g}$ for $10 \mathrm{~min}$ at room temperature. The supernatant containing mononuclear cells (MNCs) was collected, washed with PBST, and centrifuged at $300 \times \mathrm{g}$ for $10 \mathrm{~min}$ at room temperature. Further, EasySep ${ }^{\mathrm{TM}}$ Human CD8+T Cell Isolation Kit (Stemcell Technologies, catalog \#17953) was utilized to isolate highly purified CD8+T cells according to the manufacturer's guidance. The isolated cells were phenotypically identified by flow cytometry using anti-human CD3 antibody, clone UCHT1 (Stemcell Technologies, catalog \#60011) and anti-human CD8a antibody, clone RPA-T8 (Stemcell Technologies, catalog \#60022). The activity of $\mathrm{CD} 8+\mathrm{T}$ cells was measured by trypan blue staining. CD8+T cells were cultured with ImmunoCult $\mathrm{t}^{\mathrm{TM}}-\mathrm{XF} T$ 
Cell Expansion Medium (Stemcell Technologies, catalog \#10981) and stimulated with ImmunoCult ${ }^{\text {TM }}$ Human CD3/CD28 T Cell Activator (Stemcell Technologies, Catalog \#10981).

\section{Bioinformatic analysis}

To explore downstream targets of LINC01088, microRNAs expression in CRC tissues were extracted from The Tumor Cancer Genome Atlas (TCGA) dataset. In addition, the relationship between tumor G3BP1 expression and immune infiltration in colorectal cancer was analyzed using Tumor IMmune Estimation Resource (TIMER) (https://cistrome.shinyapps.io/timer/) dataset (Li et al, 2016; Li et al, 2017).

\section{Statistical analysis}

Statistical analysis was performed using SPSS 21.0 software. Graphs were made using the GraphPad Prism 8 (GraphPad Software, San Diego, CA). All data were expressed using mean \pm standard deviation and data conforming to normal distribution were compared between two groups using two-tailed Student t-test. For multi-group comparisons, statistical significance was determined by one-way analysis of variance (ANOVA) followed by Fisher's Least Significant Difference (LSD) test. Kaplan-Meier method was used to analyze the relationship between gene expression and prognosis of CRC patients. A $P<0.05$ was considered as statistically significant. ${ }^{\star} P<0.05,{ }^{\star} P P<0.01,{ }^{\star \star \star} P<0.001$, and NS: not significant.

\section{Results}

\section{LINC01088 is overexpressed in colorectal cancer and associated with adverse outcome}

We firstly collected 36 paired CRC tissues and adjacent normal tissues. qPCR results confirmed that LINC01088 expression was significantly elevated in colorectal cancer tumor tissues (Fig. 1A).

Clinicopathological analysis showed that LINC01088 expression was associated with TNM stage, metastasis, and recurrence of patients with colorectal cancer, where LINC01088 expression was higher in

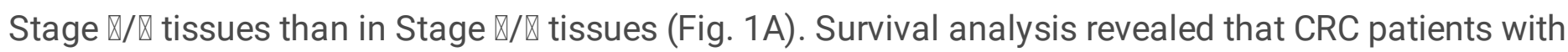
high LINC01088 levels had worse overall survival compared with CRC patients with lower levels (Fig. 1B). Besides, LINC01088 expression was also significantly higher in CRC cells compared to normal colorectal epithelial cells and LINC01088 was located in the cytoplasm ang nucleus of tumor cells (Fig. 1C, D). Consistently, fluorescence in situ hybridization (FISH) assay further identified its localization (Fig. 1E).

\section{LINC01088 knockdown significantly reduces proliferative ability of human colorectal cancer cells and patient-derived organoids}


Considering high level of LINC01088 in Caco2 and LoVo cells, these two cell lines were used to construct CRC cells with stable LINC01088 knockdown by lentiviral infection. QPCR analysis validated that both shRNAs (sh1-01088 and sh2-01088) lentivirus were able to significantly reduce LINC01088 expression levels (Fig. 2A). Cell proliferation assay and colony-formation assay confirmed that proliferative and colony-forming abilities were significantly attenuated after silencing of LINC01088 in CRC cells (Fig. 2BD). It seemed that the higher LINC01088 silencing efficiency, the lower CRC cell proliferative and colony forming capabilities. Results of cell cycle showed that LINC01088 knockdown contributed to a significant increase in the proportion of CRC cells in G0/G1 phase and a decline in G2/M phase (Fig. 2E, F).

Consistent with the in vitro results, subcutaneous xenograft experiments in nude mice further proved that LINC01088 knockdown significantly inhibited CRC cell growth (Fig. 2G-I).

Human colorectal cancer organoids (Fig. 3A) were identified by immunofluorescence with anti-SOX9, Cytokeratin 20, EpCAM, Desmin, and Ki-67 antibodies (Fig. 3B). Knockdown efficiency of organoids transduced with lentivirus was measured by visualizing green fluorescence. Under white light conditions, it could be seen that the LINC01088 knockdown resulted in smaller diameter and more cellular debris (Fig. $3 \mathrm{C}$ ), and PI staining suggested that more PI-staining positive cells were found in the organoid after LINC01088 knockdown (Fig. 3D).

\section{LINC01088 knockdown suppresses migration, invasion, and immune escape of CRC cells}

Next, we investigated the role of LINC01088 in migration and invasion of CRC cells. Transwell assays revealed that LINC01088 knockdown significantly reduced migration and invasion of Caco2 and LoVo cells (Fig. 4A-B). The higher LINC01088 silencing efficiency, the lower migratory and invasive capacities of the cells. This result was further reproduced in xenograft mouse model. Specially, the number and the lesion surface of the lung metastasis nodules are significantly reduced in LINC01088-knockdown groups (Fig. 4C-E). Survival analysis showed that silencing of LINC01088 significantly prolonged the survival of nude mice (Fig. 4F).

Given that tumor immune escape plays an important role in tumorigenesis and cancer progression, CRC cells were co-cultured with activated human CD8+T cells. After co-culturing with CD8+T cells for 12 hours, results of white light image and crystal violet staining showed that the density of adherent CRC cells with LINC01088 silencing was markedly reduced compared to the control, which was dependent on the number of CD8+T cells (Fig. 4G-J). It was suggested that LINC01088-knockdown CRC cells were more sensitive to $\mathrm{CD} 8+\mathrm{T}$ cell-mediated tumor killing relative to WT CRC cells. Similar results were observed in PI staining (Fig. 4K, L).

\section{LINC01088 exerts its biological activity by directly binding to intracellular microRNAs}

Page $11 / 21$ 
Long non-coding RNAs acts as competing endogenous RNAs (ceRNAs) to exhibit its activity when they localized in the cytoplasm. In this study, LINC01088 was mainly localized in the cytoplasm. Hence, we suspected that LINC01088 exerts its biological function via IncRNA-microRNA (miRNA)-messenger RNA (mRNA)-ceRNA networks. Therefore, microRNAs (miRNAs) that were significantly downregulated in colorectal cancer and predicted to bind directly to LINC01088 were analyzed by bioinformatics methods. A list of 35 miRNAs was obtained (Fig. 5A). And we further confirmed that 20 miRNAs were able to be directly down-regulated by LINC01088 in colorectal cancer by RIP assay (Fig. 5B). The expression levels of these 20 miRNAs were examined in LoVo cells with LINC01088 knockdown, and miRNAs that were upregulated after silencing LINC01088 were taken into consideration (Fig. 5C, D). Finally, 9 miRNAs were selected as targets for further analysis (Fig. 5E). Next, specific inhibitors of miRNAs were transfected into LINC01088-knockdown CRC cells to determine the primary downstream targets. Since miR-548b-5p and miR-548c-5p inhibitors could reverse phenotypes induced by LINC01088 knockdown, miR-548b-5p and miR-548c-5p were identified as potentially important downstream targets of LINC01088 (Fig. 6A-D). dualluciferase assay experiments further confirmed that miR-548b-5p and miR-548c-5p were indeed direct targets of LINC01088 (Fig. 6E-H).

\section{LINC01088 targets miR-548b-5p and miR-548c-5p, regulating G3BP1 expression}

MicroRNAs usually regulate mRNA expression by directly binding to them. By bioinformatics analysis, we searched for downstream target genes jointly predicted by miR-548b-5p and miR-548c-5p in multiple databases, respectively. miR-548b-5p had 9 target genes and miR-548c-5p had 41 target genes. By taking the intersection of these two sets of genes, we obtained a list of genes including $B R W D 1, G 3 B P 1$, CCDC47, DDIT4, RORA, UE4A, LY75, TRA2B (Fig. 7A). Combining survival and expression analysis in colorectal cancer, we concluded that G3BP1 may be a key downstream target of LINC01088/microRNAs axis. Ras-GTPase-activating protein SH3 domain-binding protein 1 (G3BP1) was proved to be overexpressed in a variety of cancers and targeting G3BP1 exhibited anti-tumor activity (Zhang et al, 2019). Further analysis from TCGA and TIMER databases revealed that in most human cancers including colorectal cancer G3BP1 expression is upregulated and associated with immune infiltration in colorectal cancer (Fig. S1). Next, we firstly explored G3BP1 protein expression in LINC01088-knockdown LoVo cells. Western blotting analysis showed that treatment with miR-548b-5p/ miR-548c-5p mimics contributed to a significant decrease in G3BP1 protein levels in CRC cells (Fig. 7B, C). Next, predicted binding sites between LINC01088 and miR-548b-5p (Fig. 7D), LINC01088 and miR-548c-5p (Fig. 7E) were detected by dual-luciferase reporter assay. Data revealed that three binding sites between LINC01088 and miR-548b$5 p$ are available (Fig. 7F) and two binding sites between LINC01088 and miR-548c-5p were identified (Fig. $7 G)$. 


\section{LINC01088/microRNAs/G3BP1/PD-L1 axis promotes colorectal cancer progression}

As previously described, our results suggested that LINC01088 knockdown repressed CRC cell proliferation, migration, and invasion. Subsequently, we implemented rescue experiments to verify effects of LINC01088/microRNAs/G3BP1 axis on malignant phenotypes of colorectal cancer. LINC01088 knockdown resulted in decreased G3BP1 expression at the transcriptional nor the translational levels (Fig. $8 \mathrm{~A}, \mathrm{~B})$, reduced cell viability, migratory and invasive capabilities, and weakened CD8+T cell-mediated tumor killing activity, whereas overexpression of G3BP1 could partially reverse G3BP1 expression and phenotypes caused by LINC01088 knockdown in Caco2 cells (Fig. 9C-G). Besides, when LINC01088knockdown Caco2 cells were co-cultured with activated CD8+T cells for $12 \mathrm{~h}$, G3BP1 overexpression reduced lactate dehydrogenase (LDH) levels that were significantly elevated as LINC01088 knockdown (Fig. S2). Specially, LINC01088 knockdown caused a significant decrease in PD-L1 mRNA expression and cell-surface PD-L1 expression in Caco2 cells (Fig. 8H, I). Further, tumor xenograft in nude mice revealed that G3BP1 overexpression restored LINC01088 knockdown-induced suppression of cell proliferation (Fig. 8J, K). Remarkably, LINC01088 knockdown downregulated PD-L1 expression, while G3BP1 overexpression could reverse PD-L1 expression in xenograft tumors. It implied that G3BP1 regulated PDL1 expression in CRC (Fig. 8L). These findings suggested that upregulation of LINC01088 promotes malignant phenotypes and immune escape of colorectal cancer by regulating microRNAs/G3BP1/PD-L1 axis (Fig. 9).

\section{Discussion}

Colorectal cancer is a great threat to human health. Explorations of the pathogenesis of colorectal cancer as well as clinical treatments have achieved significant progress in recent decades (Dekker et al., 2019; Nguyen et al, 2020). However, considering that long-term survival and quality of life of colorectal cancer patients have been dismal, further researches on specific mechanisms are deserved to find novel therapeutic strategies. In the present study, we found that LINC01088 was significantly upregulated in colorectal cancer tissues and LINC01088 promoted colorectal cancer progression by mediating microRNAs/G3BP1 axis.

LincRNA is one of the long non-coding RNAs (Ransohoff et al., 2018), and mounting evidence has confirmed that dysregulated LincRNA expression in tumor tissues plays an important role in multiple disease processes (Ransohoff et al., 2018; Ulitsky \& Bartel, 2013). Previous studies found that LINC01088 inhibited trophoblast cell function and led to recurrent miscarriages by activating MAPK signaling pathway (Zhao et al, 2021). Work in lung squamous cell carcinoma (LSqCC) demonstrated that through analyzing transcriptome profiling of 1771 lincRNAs in the TCGA database from 549 samples of 501 LSqCC patients, 10 lincRNAs including LINC01088 were confirmed to be significant highly-expressed risk candidates and were associated with poor prognosis (Liu et al., 2019). Another recent study has identified that LINC01088 directly binds to EZH2 inhibiting the expression of p21, a well-known oncogenic factor, 
thereby suppressing proliferation of lung cancer cells (Liu et al., 2020). Similarly, our data demonstrated that elevated LINC01088 expression facilitated proliferation of colorectal cancer cells, contributing to colorectal cancer progression. Mechanistically, LINC01088 regulated the proliferative capacity of colorectal cancer cells by adsorbing a series of intracellular miRNAs acting as a pro-cancer factor. In contrary, there is also evidence that LINC01088 expression levels are significantly lower in ovarian cancer tissues relative to ovarian epithelial tissues (Zhang et al., 2018) and low LINC01088 expression is correlated with FIGO staging, grade and metastasis (Ai et al., 2018), where LINC01088/miR-24-1-5p/p21activated kinase 4 (PAK4) axis may be responsible for the oncogenic role of LINC01088 in ovarian epithelium (Zhang et al., 2018). LINC01088 function is differential in different tissues.

Invasive capacity (Novikov et al, 2021; Yeung \& Yang, 2017) and immune escape (Liu \& Cao, 2016; Picard et al, 2020) of cells are important factors for tumor development. Our study confirmed that LINC01088 knockdown significantly reduced migratory and invasive capabilities and facilitated immune escape of colorectal cancer cells. Specifically, LINC01088 positively regulates G3BP1 expression by directly binding to and negatively regulating miR-548b-5p and miR-548c-5p, two oncogenic factors in a variety of cancers including colorectal cancers (Pan et al, 2016; Wang et al, 2020; Xu et al, 2020). G3BP1 is an oncogenic gene and targeting G3BP1 may be a promising antitumor strategy (Zhang et al., 2019). Our study is the first time to confirm the correlation between miR-548b-5p, miR-548c-5p and G3BP1. Restoration of G3BP1 levels reversed phenotypes associated with malignancy including proliferation, migration and immune escape LINC01088 knockdown CRC cells. This may provide a potential target for colorectal cancer treatment.

In summary, our findings have confirmed that LINC01088 is significantly upregulated in colorectal cancer tissues, and directly binds to miR-548b-5P and miR-548c-5P to regulate G3BP1 and PD-L1 expression, promoting colorectal cancer progression. LINC01088 exhibited tumor-promoting property. This work provides novel insights into the pathogenesis of colorectal cancer.

\section{Declarations}

\section{Funding}

This work was supported by grants from the National Natural Science Foundation of China (Grant No. 81972806, 81802093, 82002230), Jiangsu Provincial Key Research and Development Plan (Grant No. BE2019614), Elderly Health Research Project of Jiangsu Province (Grant No. LR2021017), and Specialized Cohort Research Project of Nanjing Medical University (NMUC2020035).

\section{Ethics Statements}

All patients signed informed consent form prior to surgery, and the study was approved by the Ethics Committee of Nanjing First Hospital. All animal experiments were conducted under the approval of Animal Care and Use Committee of Nanjing First Hospital. 


\section{Data Availability Statement}

The data that support the findings of this study are available from the corresponding author upon reasonable request.

\section{Conflicts of Interest}

The authors declare no conflict of interest.

\section{References}

1. Ai H, Xie W, Xiu AH, Li N, Zhang ST, Zhang YL, Xue XO (2018) The down-regulation of long noncoding RNA LINC01088 is associated with the poor prognosis of epithelial ovarian cancer patients. European review for medical and pharmacological sciences 22: 5836-5841

2. Anania G, Resta G, Marino S, Fabbri N, Scagliarini L, Marchitelli I, Fiorica F, Cavallesco G (2019) Treatment of Colorectal Cancer: a Multidisciplinary Approach. Journal of gastrointestinal cancer 50 : 458-468

3. Chi Y, Wang D, Wang J, Yu W, Yang J (2019) Long Non-Coding RNA in the Pathogenesis of Cancers. Cells 8

4. Dekker E, Tanis PJ, Vleugels JLA, Kasi PM, Wallace MB (2019) Colorectal cancer. Lancet (London, England) 394: 1467-1480

5. Dykes IM, Emanueli C (2017) Transcriptional and Post-transcriptional Gene Regulation by Long Noncoding RNA. Genomics, proteomics \& bioinformatics 15: 177-186

6. Fang Y, Fullwood MJ (2016) Roles, Functions, and Mechanisms of Long Non-coding RNAs in Cancer. Genomics, proteomics \& bioinformatics 14: 42-54

7. Franke AJ, Skelton WP, Starr JS, Parekh H, Lee JJ, Overman MJ, Allegra C, George TJ (2019) Immunotherapy for Colorectal Cancer: A Review of Current and Novel Therapeutic Approaches. Journal of the National Cancer Institute 111: 1131-1141

8. Gutschner T, Diederichs S (2012) The hallmarks of cancer: a long non-coding RNA point of view. RNA biology 9: 703-719

9. Li B, Severson E, Pignon JC, Zhao H, Li T, Novak J, Jiang P, Shen H, Aster JC, Rodig S et al (2016) Comprehensive analyses of tumor immunity: implications for cancer immunotherapy. Genome biology 17: 174

10. Li C, Pan B, Liu X, Qin J, Wang X, He B, Pan Y, Sun H, Xu T, Xu X et al (2021) Long intergenic noncoding RNA LINC00485 exerts tumor-suppressive activity by regulating miR-581/EDEM1 axis in colorectal cancer. Aging 13: 3866-3885

11. Li J, Xia R, Liu T, Cai X, Geng G (2020) LncRNA-ATB Promotes Lung Squamous Carcinoma Cell Proliferation, Migration, and Invasion by Targeting microRNA-590-5p/NF90 Axis. DNA and cell 
biology 39: 459-473

12. Li T, Fan J, Wang B, Traugh N, Chen Q, Liu JS, Li B, Liu XS (2017) TIMER: A Web Server for Comprehensive Analysis of Tumor-Infiltrating Immune Cells. Cancer research 77: e108-e110

13. Liu J, Yao Y, Hu Z, Zhou H, Zhong M (2019) Transcriptional profiling of long-intergenic noncoding RNAs in lung squamous cell carcinoma and its value in diagnosis and prognosis. Molecular genetics \& genomic medicine 7: e994

14. Liu JQ, Feng YH, Zeng S, Zhong MZ (2020) linc01088 promotes cell proliferation by scaffolding EZH2 and repressing p21 in human non-small cell lung cancer. Life sciences 241: 117134

15. Liu Y, Cao X (2016) Immunosuppressive cells in tumor immune escape and metastasis. Journal of molecular medicine (Berlin, Germany) 94: 509-522

16. Nappi A, Berretta M, Romano C, Tafuto S, Cassata A, Casaretti R, Silvestro L, Divitiis C, Alessandrini L, Fiorica F et al (2018) Metastatic Colorectal Cancer: Role of Target Therapies and Future Perspectives. Current cancer drug targets 18: 421-429

17. Nguyen LH, Goel A, Chung DC (2020) Pathways of Colorectal Carcinogenesis. Gastroenterology 158: 291-302

18. Novikov NM, Zolotaryova SY, Gautreau AM, Denisov EV (2021) Mutational drivers of cancer cell migration and invasion. British journal of cancer 124: 102-114

19. Palazzo AF, Koonin EV (2020) Functional Long Non-coding RNAs Evolve from Junk Transcripts. Cell 183: $1151-1161$

20. Pan Y, Liang W, Zhao X, Liu L, Qing Y, Li Y (2016) miR-548b inhibits the proliferation and invasion of malignant gliomas by targeting metastasis tumor-associated protein-2. Neuroreport 27: 1266-1273

21. Picard E, Verschoor CP, Ma GW, Pawelec G (2020) Relationships Between Immune Landscapes, Genetic Subtypes and Responses to Immunotherapy in Colorectal Cancer. Frontiers in immunology 11: 369

22. Quinn JJ, Chang HY (2016) Unique features of long non-coding RNA biogenesis and function. Nature reviews Genetics 17: 47-62

23. Ransohoff JD, Wei Y, Khavari PA (2018) The functions and unique features of long intergenic noncoding RNA. Nature reviews Molecular cell biology 19: 143-157

24. Sanchez Calle A, Kawamura Y, Yamamoto Y, Takeshita F, Ochiya T (2018) Emerging roles of long non-coding RNA in cancer. Cancer science 109: 2093-2100

25. Schmitz SU, Grote P, Herrmann BG (2016) Mechanisms of long noncoding RNA function in development and disease. Cellular and molecular life sciences : CMLS73: 2491-2509

26. Sung H, Ferlay J, Siegel RL, Laversanne M, Soerjomataram I, Jemal A, Bray F (2021) Global Cancer Statistics 2020: GLOBOCAN Estimates of Incidence and Mortality Worldwide for 36 Cancers in 185 Countries. CA: a cancer journal for clinicians 71: 209-249

27. Ulitsky I, Bartel DP (2013) lincRNAs: genomics, evolution, and mechanisms. Cell 154: 26-46 
28. Van Cutsem E, Cervantes A, Nordlinger B, Arnold D (2014) Metastatic colorectal cancer: ESMO Clinical Practice Guidelines for diagnosis, treatment and follow-up. Annals of oncology : official journal of the European Society for Medical Oncology 25 Suppl 3: iii1-9

29. Wang Z, Wu X, Hou X, Zhao W, Yang C, Wan W, Chen L (2020) miR-548b-3p functions as a tumor suppressor in lung cancer. Lasers in medical science 35: 833-839

30. Xu M, Xu X, Pan B, Chen X, Lin K, Zeng K, Liu X, Xu T, Sun L, Qin J et al (2019) LncRNA SATB2-AS1 inhibits tumor metastasis and affects the tumor immune cell microenvironment in colorectal cancer by regulating SATB2. Molecular cancer 18: 135

31. Xu Y, Zhong YD, Zhao XX (2020) MiR-548b suppresses proliferative capacity of colorectal cancer by binding WNT2. European review for medical and pharmacological sciences 24: 10535-10541

32. Yan Y, Ren L, Liu Y, Liu L (2021) Long non-coding RNA CRNDE as potential biomarkers facilitate inflammation and apoptosis in alcoholic liver disease. Aging 13: 23233-23244

33. Yeung KT, Yang J (2017) Epithelial-mesenchymal transition in tumor metastasis. Molecular oncology 11: $28-39$

34. You J, Li J, Ke C, Xiao Y, Lu C, Huang F, Mi Y, Xia R, Li Q (2021) Oncogenic long intervening noncoding RNA Linc00284 promotes c-Met expression by sponging miR-27a in colorectal cancer. Oncogene 40: 4151-4166

35. Zhang CH, Wang JX, Cai ML, Shao R, Liu H, Zhao WL (2019) The roles and mechanisms of G3BP1 in tumour promotion. Journal of drug targeting 27: 300-305

36. Zhang W, Fei J, Yu S, Shen J, Zhu X, Sadhukhan A, Lu W, Zhou J (2018) LINC01088 inhibits tumorigenesis of ovarian epithelial cells by targeting miR-24-1-5p. Scientific reports 8: 2876

37. Zhao H, Li Y, Dong N, Zhang L, Chen X, Mao H, Al-Ameri SAA, Wang X, Wang Q, Du L et al (2021) LncRNA LINC01088 inhibits the function of trophoblast cells, activates the MAPK-signaling pathway and associates with recurrent pregnancy loss. Molecular human reproduction 27

38. Zhou T, Lin K, Nie J, Pan B, He B, Pan Y, Sun H, Xu T, Wang S (2021) LncRNA SPINT1-AS1 promotes breast cancer proliferation and metastasis by sponging let-7 a/b/i-5p. Pathology, research and practice 217: 153268

\section{Figures}

\section{Figure 1}

LINC01088 expression in tumor tissues of patients with colorectal cancer and its subcellular localization. (A) LINC01088 expression levels in tumor tissues and adjacent normal tissues of patients with colorectal cancer. Association analysis of LINC01088 expression and the patients' clinical characteristics including tumor-node-metastasis stages, distant metastasis and recurrence. (B) Survival analysis based on 
LINC01088 expression levels. (C) LINC01088 expression in human colorectal cancer cell lines and colonic normal epithelial cells. (D) Nucleocytoplasmic distribution of LINC01088 in CRC cells. (E) Subcellular localization of LINC01088 in cells and tissues was tested by FISH assay. Data were expressed using mean \pm standard deviation. Comparison between two groups using two-tailed Student t-test. For multigroup comparisons, statistical significance was determined by one-way ANOVA followed by Fisher's LSD test. Kaplan-Meier method was used to analyze the relationship between gene expression and prognosis of $C R C$ patients. ${ }^{*} P<0.05,{ }^{*} P<0.01,{ }^{\star} * * P<0.001$. TNM, tumor-node-metastasis; $M$, metastasis; $R$, recurrence; $\mathrm{CRC}$, colorectal cancer; FISH, RNA-fluorescence in situ hybridization.

\section{Figure 2}

Effects of LINC01088 knockdown on CRC cell proliferation in vitro and in vivo. (A) LINC01088 knockdown in CRC cells was achieved by lentivirus-mediated shRNA interference. (B) Cell viability of LINC01088knockdown CRC cells at different time points was measured by CCK-8 assay. (C, D) Colony-forming ability of LINC01088-knockdown (C) Caco2 and (D) LoVo cells was evaluated by colony formation assay after culturing for 14 consecutive days. (E, F) Cell cycle distribution of LINC01088-knockdown (E) Caco2 and (F) LoVo cells was tested by flow cytometry. (G) Tumor growth of LINC01088-knockdown xenograft tumors. (H) Image of xenograft tumors at 42 days after inoculation. (I) Tumor volume of LINC01088knockdown xenograft tumors at 42 days after inoculation. $\mathrm{N}=6$ mice per group. Comparisons among groups were analyzed by one-way ANOVA followed by Fisher's LSD test. ${ }^{*} P<0.05,{ }^{\star *} P<0.01,{ }^{\star} * \star P<0.001$. $\mathrm{CRC}$, colorectal cancer.

\section{Figure 3}

Cultivation, identification and lentivirus infection of human colorectal cancer organoids. (A) Organoids at P0 and P1. (B) Immunofluorescence staining for organoids using anti-SOX9, Cytokeratin 20, EpCAM, Desmin, and Ki-67 antibodies. (C) Organoids at P1 were infected with LINC01088-knckdown and control lentivirus. (D) Organoids infected with lentivirus were stained with PI $(1 \mu \mathrm{g} / \mathrm{ml})$ for $5 \mathrm{~min}$. Green indicates GFP fluorescence; and red, PI fluorescence.

\section{Figure 4}

Effects of LINC01088 knockdown on the migration and invasion, lung metastatic ability of CRC cells in vitro and in vivo. (A, B) Migratory and invasive capabilities of LINC01088 knockdown (A) Caco2 and (B) LoVo cells were tested by transwell assays. (C) Schematic illustration of animal experiments. (D) Representative images and quantification of lung metastatic nodules in xenograft mouse model. (E) HE 
staining of lung metastatic nodules. (F) Survival curves of CRC-bearing nude mice. (G, H) After coculturing for $12 \mathrm{~h}$, LINC01088-knockdown CRC cells and CD8+T cells were imaged under the bright field. $(\mathbf{l}, \mathbf{J})$ After co-culturing for $12 \mathrm{~h}$, surviving CRC cells were stained with crystal violet. $(\mathrm{K}, \mathrm{L})$ After coculturing for $12 \mathrm{~h}$, apoptotic CRC cells were stained by PI. Data were expressed using mean \pm standard deviation. Comparison between two groups using two-tailed Student t-test. For multi-group comparisons, statistical significance was determined by one-way ANOVA followed by Fisher's LSD test. ${ }^{*} P<0.05$, ${ }^{\star} P<0.01,{ }^{\star \star *} P<0.001$. HE, haematoxylin-eosin staining; PI, propidium iodide. CRC, colorectal cancer.

\section{Figure 5}

Determination of the downstream targets of LINC01088 in colorectal cancer. (A) Screening process of the downstream targets of LINC01088. (B) RIP assay was performed to validate the relationship between microRNA and Linc01088 in Caco2 cells. (C, D) The expression levels of 20 microRNAs in (C) sh1LINC01088 Caco2 cells and (D) sh2-LINC01088 Caco2 cells. (E) Nine differentially expressed microRNAs that interacted directly with LINC01088 in Caco2 cells. Data were expressed using mean \pm standard deviation. Comparison between two groups using two-tailed Student t-test. For multi-group comparisons, statistical significance was determined by one-way ANOVA followed by Fisher's LSD test. ${ }^{*} P<0.05$. RIP, RNA immunoprecipitation assay.

\section{Figure 6}

miR-548b-5p and miR-548c-5p are the main direct downstream targets of LINC01088 in colorectal cancer. (A) Cell viability of LINC01088-knockdown Caco2 cells transfected with microRNA inhibitor for $48 \mathrm{~h}$. (B) Representative images and statistical analysis of the migration and invasion of LINC01088-knockdown Caco 2 cells transfected with microRNA inhibitor. (C) After co-culturing for 12 h, LINC01088-knockdown Caco2 cells transfected with microRNA inhibitor were stained with crystal-violet. (D) Two microRNAs were determined according to the Venn diagram. (E, G) Predicted binding sites of (E) miR-548b-5p or (G) miR548c-5p on LINC01088 sequence. $(F, H)$ The interaction between LINC01088 and $(F)$ miR-548b-5p or $(H)$ miR-548c-5p was determined by dual-luciferase reporter system analysis. Data were expressed using mean \pm standard deviation. Comparisons among groups was analyzed by one-way ANOVA followed by Fisher's LSD test. ${ }^{*} P<0.05,{ }^{*} P<0.01, * \star \star * P<0.001, \mathrm{NS}$, not significant. 
G3BP1 is the common direct downstream target of miR-548b-5p and miR-548c-5p in colorectal cancer. (A) Screening process of the common downstream targets of miR-548b-5p and miR-548c-5p. (B, C) G3BP1 protein expression in Caco2 cells after transfection with (B) miR-548b-5p or (C) miR-548c-5p mimics for 48 h. (D, E) Predicted binding sites between G3BP1 mRNA and (D) miR-548b-5p or (E) miR548c-5p. (F, G) The interaction between G3BP1 mRNA and (F) miR-548b-5p or (G) miR-548c-5p was determined by dual-luciferase reporter system analysis. Data were expressed using mean \pm standard deviation. Comparison between two groups using two-tailed Student t-test. Comparisons among groups was analyzed by one-way ANOVA followed by Fisher's LSD test. ${ }^{*} P<0.05, * \star P<0.01, * \star \star P<0.001$, NS, not significant.

\section{Figure 8}

Effects of LINC01088/microRNAs/G3BP1axis on colorectal cancer. (A, B) The (A) mRNA and (B) protein expression levels of G3BP1 in LINC01088-knockdown Caco2 cells. (C, D) The (C) mRNA and (D) protein expression levels of G3BP1 in LINC01088-knockdown Caco2 cells infected with or without G3BP1-

overexpressing lentivirus. (E) Cell viability was evaluated by CCK-8 assay. (F) Cell migratory and invasive capabilities were tested by transwell assays. (G) After co-culturing with CD8+T cells for $12 \mathrm{~h}$, surviving Caco2 cells were stained with crystal-violet. (H) PD-L1 mRNA expression in LINC01088-knockdown CRC cells. (I) PD-L1 cell-surface expression was assessed by flow cytometry. (J) Growth curves of xenograft tumors. (K) Tumor volume of xenograft tumors at 36 days after inoculation. (L) PD-L1 mRNA expression in xenograft tumors ( $n=3$ per group). Data were expressed using mean \pm standard deviation. Comparison between two groups using two-tailed Student t-test. Comparisons among groups was analyzed by oneway ANOVA followed by Fisher's LSD test. ${ }^{\star \star} P<0.01,{ }^{\star}{ }^{* \star} P<0.001$.

\section{Figure 9}

Effects of LINC01088/microRNAs/G3BP1axis on colorectal cancer. (A, B) The (A) mRNA and (B) protein expression levels of G3BP1 in LINC01088-knockdown Caco2 cells. (C, D) The (C) mRNA and (D) protein expression levels of G3BP1 in LINC01088-knockdown Caco2 cells infected with or without G3BP1overexpressing lentivirus. (E) Cell viability was evaluated by CCK-8 assay. (F) Cell migratory and invasive capabilities were tested by transwell assays. (G) After co-culturing with CD8+T cells for $12 \mathrm{~h}$, surviving Caco2 cells were stained with crystal-violet. (H) PD-L1 mRNA expression in LINC01088-knockdown CRC cells. (I) PD-L1 cell-surface expression was assessed by flow cytometry. (J) Growth curves of xenograft tumors. (K) Tumor volume of xenograft tumors at 36 days after inoculation. (L) PD-L1 mRNA expression 
in xenograft tumors ( $\mathrm{n}=3$ per group). Data were expressed using mean \pm standard deviation. Comparison between two groups using two-tailed Student t-test. Comparisons among groups was analyzed by oneway ANOVA followed by Fisher's LSD test. ${ }^{\star \star} P<0.01, * \star \star P<0.001$.

\section{Supplementary Files}

This is a list of supplementary files associated with this preprint. Click to download.

- Supplementaryfigure1.tif

- Supplementaryfigure2.tif 